$\mathrm{J}$ o u r n a $\mathrm{l}$ of

Mathematics

and Applications

JMA No 37, pp 67-84 (2014)

\title{
Properties of higher order differential polynomials generated by solutions of complex differential equations in the unit disc
}

\author{
Zinelâabidine Latreuch, Benharrat Belaïdi
}

ABstraCt: The main purpose of this paper is to study the controllability of solutions of the differential equation

$$
f^{(k)}+A_{k-1}(z) f^{(k-1)}+\cdots+A_{1}(z) f^{\prime}+A_{0}(z) f=0 .
$$

In fact, we study the growth and oscillation of higher order differential polynomial with meromorphic coefficients in the unit disc $\Delta=$ $\{z:|z|<1\}$ generated by solutions of the above $k^{t h}$ order differential equation.

AMS Subject Classification: 34M10, 30 D35.

Keywords and Phrases: Iterated p-order, Linear differential equations, Iterated exponent of convergence of the sequence of distinct zeros, Unit disc.

\section{Introduction and main results}

Throughout this paper, we assume that the reader is familiar with the fundamental results and the standard notations of the Nevanlinna's value distribution theory on the complex plane and in the unit disc $\Delta=\{z:|z|<1\}$ (see [13], [14], [18], [20]). We need to give some definitions and discussions. Firstly, let us give two definitions about the degree of small growth order of functions in $\Delta$ as polynomials on the complex plane $\mathbb{C}$. There are many types of definitions of small growth order of functions in $\Delta$ (see $[10],[11])$.

Definition 1.1 ([10], [11]) Let $f$ be a meromorphic function in $\Delta$, and

$$
D(f):=\limsup _{r \rightarrow 1^{-}} \frac{T(r, f)}{\log \frac{1}{1-r}}=b .
$$

COPYRIGHT (c) by Publishing Department Rzeszów University of Technology P.O. Box 85, 35-959 Rzeszów, Poland 
If $b<\infty$, we say that $f$ is of finite $b$ degree (or is non-admissible). If $b=\infty$, we say that $f$ is of infinite degree (or is admissible), both defined by characteristic function $T(r, f)$.

Definition $1.2([10],[11])$ Let $f$ be an analytic function in $\Delta$, and

$$
\left.D_{M}(f):=\limsup _{r \rightarrow 1^{-}} \frac{\log ^{+} M(r, f)}{\log \frac{1}{1-r}}=a<\infty \quad \text { or } a=\infty\right),
$$

then we say that $f$ is a function of finite $a$ degree (or of infinite degree) defined by maximum modulus function $M(r, f)=\max _{|z|=r}|f(z)|$.

Now we give the definitions of iterated order and growth index to classify generally the functions of fast growth in $\Delta$ as those in $\mathbb{C}$ (see [4], [17], [18]). Let us define inductively, for $r \in[0,1)$, $\exp _{1} r=e^{r}$ and $\exp _{p+1} r=\exp \left(\exp _{p} r\right), p \in \mathbb{N}$. We also define for all $r$ sufficiently large in $(0,1), \log _{1} r=\log r$ and $\log _{p+1} r=\log \left(\log _{p} r\right), p \in$ $\mathbb{N}$. Moreover, we denote by $\exp _{0} r=r, \log _{0} r=r$, $\exp _{-1} r=\log _{1} r, \log _{-1} r=\exp _{1} r$.

Definition $1.3[5,6]$ The iterated $p$-order of a meromorphic function $f$ in $\Delta$ is defined by

$$
\rho_{p}(f)=\limsup _{r \rightarrow 1^{-}} \frac{\log _{p}^{+} T(r, f)}{\log \frac{1}{1-r}}(p \geq 1) .
$$

For an analytic function $f$ in $\Delta$, we also define

$$
\rho_{M, p}(f)=\limsup _{r \rightarrow 1^{-}} \frac{\log _{p+1}^{+} M(r, f)}{\log \frac{1}{1-r}}(p \geq 1) .
$$

Remark 1.1 It follows by M. Tsuji in [25] that if $f$ is an analytic function in $\Delta$, then

$$
\rho_{1}(f) \leq \rho_{M, 1}(f) \leq \rho_{1}(f)+1
$$

However, it follows by Proposition 2.2.2 in [18]

$$
\rho_{M, p}(f)=\rho_{p}(f),(p \geq 2) .
$$

Definition 1.4 [5] The growth index of the iterated order of a meromorphic function $f(z)$ in $\Delta$ is defined by

$$
i(f)=\left\{\begin{array}{cc}
0, & \text { if } f \text { is non-admissible, } \\
\min \left\{j \in \mathbb{N}, \rho_{j}(f)<\infty\right\}, & \text { if } f \text { is admissible, } \\
+\infty, & \text { if } \rho_{j}(f)=\infty \text { for all } j \in \mathbb{N} .
\end{array}\right.
$$

For an analytic function $f$ in $\Delta$, we also define

$$
i_{M}(f)=\left\{\begin{array}{cc}
0, & \text { if } f \text { is non-admissible, } \\
\min \left\{j \in \mathbb{N}, \rho_{M, j}(f)<\infty\right\}, & \text { if } f \text { is admissible, } \\
+\infty, & \text { if } \rho_{M, j}(f)=\infty \text { for all } j \in \mathbb{N} .
\end{array}\right.
$$


Definition 1.5 $[3,15,20]$ The iterated $p$-type of a meromorphic function $f$ of iterated $p$-order $\rho(0<\rho<\infty)$ in $\Delta$ is defined by

$$
\tau_{p}(f)=\limsup _{r \rightarrow 1^{-}}(1-r)^{\rho_{p}(f)} \log _{p-1}^{+} T(r, f) .
$$

Definition 1.6 [7] Let $f$ be a meromorphic function in $\Delta$. Then the iterated $p-$ convergence exponent of the sequence of zeros of $f(z)$ is defined by

$$
\lambda_{p}(f)=\limsup _{r \rightarrow 1^{-}} \frac{\log _{p}^{+} N\left(r, \frac{1}{f}\right)}{\log \frac{1}{1-r}},
$$

where $N\left(r, \frac{1}{f}\right)$ is the counting function of zeros of $f(z)$ in $\{z \in \mathbb{C}:|z| \leq r\}$. Similarly, the iterated $p$-convergence exponent of the sequence of distinct zeros of $f(z)$ is defined by

$$
\bar{\lambda}_{p}(f)=\limsup _{r \rightarrow 1^{-}} \frac{\log _{p}^{+} \bar{N}\left(r, \frac{1}{f}\right)}{\log \frac{1}{1-r}}
$$

where $\bar{N}\left(r, \frac{1}{f}\right)$ is the counting function of distinct zeros of $f(z)$ in $\{z \in \mathbb{C}:|z| \leq r\}$.

Definition 1.7 [7] The growth index of the convergence exponent of the sequence of the zeros of $f(z)$ in $\Delta$ is defined by

$$
i_{\lambda}(f)=\left\{\begin{array}{cc}
0, & \text { if } N\left(r, \frac{1}{f}\right)=O\left(\log \frac{1}{1-r}\right), \\
\min \left\{j \in \mathbb{N}, \lambda_{j}(f)<\infty\right\}, & \text { if some } j \in \mathbb{N} \text { with } \lambda_{j}(f)<\infty, \\
+\infty, & \text { if } \lambda_{j}(f)=\infty \text { for all } j \in \mathbb{N} .
\end{array}\right.
$$

Similarly, we can define the growth index of the convergence exponent of the sequence of distinct zeros $i_{\bar{\lambda}}(f)$ of $f(z)$ in $\Delta$.

Consider the complex differential equation

$$
f^{(k)}+A_{k-1}(z) f^{(k-1)}+\cdots+A_{1}(z) f^{\prime}+A_{0}(z) f=0
$$

and the $k^{\text {th }}$ order differential polynomial

$$
g_{k}=d_{k} f^{(k)}+d_{k-1} f^{(k-1)}+\cdots+d_{0} f,
$$

where $A_{j}(j=0,1, \cdots, k-1)$ and $d_{i}(i=0,1, \cdots, k)$ are meromorphic functions in $\Delta$.

Let $\mathcal{L}(\mathbf{G})$ denote a differential subfield of the field $\mathcal{M}(\mathbf{G})$ of meromorphic functions in a domain $\mathbf{G} \subset \mathbb{C}$. If $\mathbf{G}=\boldsymbol{\Delta}$, we simply denote $\mathcal{L}$ instead of $\mathcal{L}(\boldsymbol{\Delta})$. Special case of such differential subfield

$$
\mathcal{L}_{p+1, \rho}=\left\{g \text { meromorphic: } \rho_{p+1}(g)<\rho\right\},
$$


where $\rho$ is a positive constant. In [7], T. B. Cao, H. Y. Xu and C. X. Zhu studied the complex oscillation of differential polynomial generated meromorphic solutions of second order linear differential equations with meromorphic coefficients and obtained the following results.

Theorem A [7] Let $A$ be an admissible meromorphic function of finite iterated order $\rho_{p}(A)=\rho>0(1 \leq p<\infty)$ in the unit disc $\Delta$ such that $\delta(\infty, A)=\liminf _{r \rightarrow 1^{-}} \frac{m(r, A)}{T(r, A)}=$ $\delta>0$, and let $f$ be a non-zero meromorphic solution of the differential equation

$$
f^{\prime \prime}+A(z) f=0,
$$

such that $\delta(\infty, f)>0$. Moreover, let

$$
P[f]=\sum_{j=0}^{k} p_{j} f^{(j)}
$$

be a linear differential polynomial with coefficients $p_{j} \in \mathcal{L}_{p+1, \rho}$, assuming that at least one of the coefficients $p_{j}$ does not vanish identically. If $\varphi \in \mathcal{L}_{p+1, \rho}$ is a non-zero meromorphic function in $\Delta$, and neither $P[f]$ nor $P[f]-\varphi$ vanishes identically, then we have

$$
i(f)=i_{\bar{\lambda}}(P[f]-\varphi)=p+1
$$

and

$$
\bar{\lambda}_{p}(P[f]-\varphi)=\rho_{p+1}(f)=\rho_{p}(A)=\rho
$$

if $p>1$, while

$$
\rho_{p}(A) \leq \bar{\lambda}_{p+1}(P[f]-\varphi) \leq \rho_{p+1}(f) \leq \rho_{p}(A)+1
$$

if $p=1$.

Remark 1.2 The idea of the proofs of Theorem A is borrowed from the paper of Laine, Rieppo [19] with the modifications reflecting the change from the complex plane $\mathbb{C}$ to the unit disc $\boldsymbol{\Delta}$.

Before we state our results, we define the sequence of meromorphic functions $\alpha_{i, j}$ $(j=0, \cdots, k-1)$ in $\Delta$ by

$$
\alpha_{i, j}=\left\{\begin{array}{c}
\alpha_{i, j-1}^{\prime}+\alpha_{i-1, j-1}-A_{i} \alpha_{k-1, j-1}, \text { for all } i=1, \cdots, k-1, \\
\alpha_{0, j-1}^{\prime}-A_{0} \alpha_{k-1, j-1}, \text { for } i=0
\end{array}\right.
$$

and

$$
\alpha_{i, 0}=d_{i}-d_{k} A_{i}, \text { for } i=0, \cdots, k-1
$$

we define also $h_{k}$ by

$$
h_{k}=\left|\begin{array}{ccccc}
\alpha_{0,0} & \alpha_{1,0} & \cdot & \cdot & \alpha_{k-1,0} \\
\alpha_{0,1} & \alpha_{1,1} & \cdot & \cdot & \alpha_{k-1,1} \\
\cdot & \cdot & \cdot & \cdot & \cdot \\
\cdot & \cdot & \cdot & \cdot & \cdot \\
\alpha_{0, k-1} & \alpha_{1, k-1} & \cdot & \cdot & \alpha_{k-1, k-1}
\end{array}\right|
$$


and $\psi_{k}(z)$ by

$$
\psi_{k}(z)=C_{0} \varphi+C_{1} \varphi^{\prime}+\cdots+C_{k-1} \varphi^{(k-1)},
$$

where $C_{j}(j=0, \cdots, k-1)$ are finite iterated $p$-order meromorphic functions in $\Delta$ depending on $\alpha_{i, j}$, and $\varphi \neq \equiv$ is a meromorphic function in $\Delta$ with $\rho_{p}(\varphi)<\infty$.

The main purpose of this paper is to study the controllability of solutions of the differential equation (1.1). In the fact we study the growth and oscillation of higher order differential polynomial with meromorphic coefficients in the unit disc $\Delta$ generated by solutions of equation (1.1).

Theorem 1.1 Let $A_{i}(z)(i=0,1, \cdots, k-1)$ be meromorphic functions in $\Delta$ of finite iterated $p$-order. Let $d_{j}(z)(j=0,1, \cdots, k)$ be finite iterated $p$-order meromorphic functions in $\Delta$ that are not all vanishing identically such that $h \not \equiv 0$. If $f(z)$ is an infinite iterated $p$-order meromorphic solution in $\Delta$ of $(1.1)$ with $\rho_{p+1}(f)=\rho$, then the differential polynomial (1.2) satisfies

$$
\rho_{p}\left(g_{k}\right)=\rho_{p}(f)=\infty
$$

and

$$
\rho_{p+1}\left(g_{k}\right)=\rho_{p+1}(f)=\rho .
$$

Furthermore, if $f$ is a finite iterated $p$-order meromorphic solution in $\Delta$ such that

$$
\rho_{p}(f)>\max \left\{\rho_{p}\left(A_{i}\right)(i=0,1, \cdots, k-1), \rho_{p}\left(d_{j}\right) \quad(j=0,1, \cdots, k)\right\},
$$

then

$$
\rho_{p}\left(g_{k}\right)=\rho_{p}(f) .
$$

Remark 1.3 In Theorem 1.1, if we do not have the condition $h \not \equiv 0$, then the conclusions of Theorem 1.1 cannot hold. For example, if we take $d_{i}=d_{k} A_{i}$ $(i=0, \cdots, k-1)$, then $h \equiv 0$. It follows that $g_{k} \equiv 0$ and $\rho_{p}\left(g_{k}\right)=0$. So, if $f(z)$ is an infinite iterated $p$-order meromorphic solution of $(1.1)$, then $\rho_{p}\left(g_{k}\right)=0<\rho_{p}(f)=$ $\infty$, and if $f$ is a finite iterated $p$-order meromorphic solution of (1.1) such that (1.5) holds, then $\rho_{p}\left(g_{k}\right)=0<\rho_{p}(f)$.

Theorem 1.2 Under the hypotheses of Theorem 1.1, let $\varphi(z) \not \equiv 0$ be a meromorphic function in $\Delta$ with finite iterated $p$-order such that $\psi_{k}(z)$ is not a solution of (1.1). If $f(z)$ is an infinite iterated p-order meromorphic solution in $\Delta$ of (1.1) with $\rho_{p+1}(f)=\rho$, then the differential polynomial (1.2) satisfies

$$
\bar{\lambda}_{p}\left(g_{k}-\varphi\right)=\lambda_{p}\left(g_{k}-\varphi\right)=\rho_{p}(f)=\infty
$$

and

$$
\bar{\lambda}_{p+1}\left(g_{k}-\varphi\right)=\lambda_{p+1}\left(g_{k}-\varphi\right)=\rho .
$$

Furthermore, if $f$ is a finite iterated $p$-order meromorphic solution in $\Delta$ such that

$$
\rho_{p}(f)>\max \left\{\rho_{p}\left(A_{i}\right)(i=0,1, \cdots, k-1), \rho_{p}\left(d_{j}\right) \quad(j=0,1, \cdots, k), \rho_{p}(\varphi)\right\},
$$


then

$$
\bar{\lambda}_{p}\left(g_{k}-\varphi\right)=\lambda_{p}\left(g_{k}-\varphi\right)=\rho_{p}(f) .
$$
in $[23]$.

From Theorems 1-2, we obtain the following corollaries which have been proved

Corollary $1.1[23]$ Suppose that $A(z)$ is admissible meromorphic function in $\Delta$ such that $i(A)=p(1 \leq p<\infty)$ and $\delta(\infty, A)=\delta>0$. Let $d_{j}(z)(j=0,1, \cdots, k)$ be finite iterated $p$-order meromorphic functions in $\Delta$ that are not all vanishing identically such that $h \neq \equiv$, and let $f$ be a nonzero meromorphic solution of

$$
f^{(k)}+A(z) f=0 .
$$

If $\delta(\infty, f)>0$, then the differential polynomial $g_{k}$ satisfies $i\left(g_{k}\right)=p+1$ and $\rho_{p+1}\left(g_{k}\right)=\rho_{p+1}(f)=\rho_{p}(A)$ if $p>1$, while

$$
\rho_{p}(A) \leq \rho_{p+1}\left(g_{k}\right)=\rho_{p+1}(f) \leq \rho_{p}(A)+1
$$

if $p=1$.

Corollary $1.2[23]$ Under the hypotheses of Corollary 1.1, let $\varphi(z) \not \equiv 0$ be meromorphic function in $\Delta$ with finite iterated $p$-order such that $\psi_{k}(z) \not \equiv 0$. Then the differential polynomial (1.2) satisfies

$$
\bar{\lambda}_{p+1}\left(g_{k}-\varphi\right)=\lambda_{p+1}\left(g_{k}-\varphi\right)=\rho_{p+1}(f)=\rho_{p}(A)
$$

if $p>1$, while

$$
\rho_{p}(A) \leq \bar{\lambda}_{p+1}\left(g_{k}-\varphi\right)=\lambda_{p+1}\left(g_{k}-\varphi\right)=\rho_{p+1}(f) \leq \rho_{p}(A)+1
$$

if $p=1$.

Remark 1.4 The present article may be understood as an extension and improvement of the recent article of the authors [23] from equation (1.7) to equation (1.1). The method used in the proofs of our theorems is simple and quite different from the method used in the papers of Laine and Rieppo [19] and Cao, Xu and Zhu [7] .

We consider now the differential equation

$$
f^{\prime \prime}+A_{1}(z) f^{\prime}+A_{0}(z) f=0
$$

where $A_{1}(z), A_{0}(z)$ are analytic functions of finite iterated $p$-order in the unit disc $\Delta$. In the following we will give sufficient conditions on $A_{1}$ and $A_{0}$ which satisfied the results of Theorem 1.1 and Theorem 1.2 without the conditions " $h_{k} \not \equiv 0$ " and " $\psi_{k}(z)$ is not a solution of (1.1) " where $k=2$.

Corollary 1.3 Let $A_{1}(z), A_{0}(z)(\not \equiv 0)$ be analytic functions in $\Delta$ such that $\rho_{p}\left(A_{0}\right)=$ $\rho(0<\rho<\infty), \tau_{p}\left(A_{0}\right)=\tau(0<\tau<\infty)$, and let $\rho_{p}\left(A_{1}\right)<\rho_{p}\left(A_{0}\right)$ or $\tau_{p}\left(A_{1}\right)<$ 
$\tau_{p}\left(A_{0}\right)$ if $\rho_{p}\left(A_{0}\right)=\rho_{p}\left(A_{1}\right)$. Let $d_{2}, d_{1}, d_{0}$ be analytic functions in $\Delta$ such that at least one of $d_{2}, d_{1}, d_{0}$ does not vanish identically with $\max \left\{\rho_{p}\left(d_{j}\right) \quad(j=0,1,2)\right\}<$ $\rho_{p}\left(A_{0}\right)$. If $f \not \equiv 0$ is a solution of $(1.8)$, then the differential polynomial $g_{2}=d_{2} f^{\prime \prime}+$ $d_{1} f^{\prime}+d_{0} f$ satisfies $\rho_{p+1}\left(g_{2}\right)=\rho_{p+1}(f)=\rho_{p}\left(A_{0}\right)$ if $p>1$, while

$$
\rho_{p}\left(A_{0}\right) \leq \rho_{p+1}\left(g_{2}\right)=\rho_{p+1}(f) \leq \max \left\{\rho_{M}\left(A_{j}\right)(j=0,1)\right\}
$$

if $p=1$.

Corollary 1.4 Let $A_{1}(z), A_{0}(z)(\not \equiv 0)$ be analytic functions in $\Delta$ such that $\rho_{p}\left(A_{0}\right)=$ $\rho(0<\rho<\infty), \tau_{p}\left(A_{0}\right)=\tau(0<\tau<\infty)$, and let $\rho_{p}\left(A_{1}\right)<\rho_{p}\left(A_{0}\right)$ or $2 \tau_{p}\left(A_{1}\right)<$ $\tau_{p}\left(A_{0}\right)$ if $\rho_{p}\left(A_{0}\right)=\rho_{p}\left(A_{1}\right)$. Let $d_{2}, d_{1}, d_{0}$ be analytic functions in $\Delta$ such that at least one of $d_{2}, d_{1}, d_{0}$ does not vanish identically with $\max \left\{\rho_{p}\left(d_{j}\right)(j=0,1,2)\right\}<$ $\rho_{p}\left(A_{1}\right)$, and let $\varphi(z) \not \equiv 0$ be analytic function in $\Delta$ of finite iterated $p$-order such that $\psi_{2}(z) \not \equiv 0$. If $f \not \equiv 0$ is a solution of (1.8), then the differential polynomial $g_{2}=d_{2} f^{\prime \prime}+d_{1} f^{\prime}+d_{0} f$ satisfies

$$
\bar{\lambda}_{p+1}\left(g_{2}-\varphi\right)=\lambda_{p+1}\left(g_{2}-\varphi\right)=\rho_{p+1}(f)=\rho_{p}\left(A_{0}\right)
$$

if $p>1$, while

$$
\rho_{p}\left(A_{0}\right) \leq \bar{\lambda}_{p+1}\left(g_{2}-\varphi\right)=\lambda_{p+1}\left(g_{2}-\varphi\right)=\rho_{p+1}(f) \leq \max \left\{\rho_{M}\left(A_{j}\right) \quad(j=0,1)\right\}
$$

if $p=1$.

Remark 1.5 For some papers related in the complex plane see $[19,22,24]$ and in the unit disc see $[7,9,12]$.

\section{Auxiliary lemmas}

Lemma $2.1[8]$ Let $A_{0}, A_{1}, \cdots, A_{k-1}, F \not \equiv 0$ be meromorphic functions in $\Delta$, and let $f$ be a meromorphic solution of the differential equation

$$
f^{(k)}+A_{k-1}(z) f^{(k-1)}+\cdots+A_{1}(z) f^{\prime}+A_{0}(z) f=F(z)
$$

such that $i(f)=p(1 \leq p<\infty)$. If either

$$
\max \left\{i\left(A_{j}\right)(j=0,1, \cdots, k-1), i(F)\right\}<p
$$

or

$$
\max \left\{\rho_{p}\left(A_{j}\right)(j=0,1, \cdots, k-1), \rho_{p}(F)\right\}<\rho_{p}(f),
$$

then

$$
i_{\bar{\lambda}}(f)=i_{\lambda}(f)=i(f)=p
$$

and

$$
\bar{\lambda}_{p}(f)=\lambda_{p}(f)=\rho_{p}(f) .
$$


Using the same arguments as in the proof of Lemma 2.1 (see, the proof of Lemma 2.5 in $[8]$ ), we easily obtain the following lemma.

Lemma 2.2 Let $A_{0}, A_{1}, \cdots, A_{k-1}, F \not \equiv 0$ be finite iterated $p$-order meromorphic functions in the unit disc $\Delta$. If $f$ is a meromorphic solution with $\rho_{p}(f)=\infty$ and $\rho_{p+1}(f)=\rho<\infty$ of equation $(2.1)$, then $\bar{\lambda}_{p}(f)=\lambda_{p}(f)=\rho_{p}(f)=\infty$ and $\bar{\lambda}_{p+1}(f)=\lambda_{p+1}(f)=\rho_{p+1}(f)=\rho$.

Lemma 2.3 [5] Let $p \geq 1$ be an integer, and let $A_{0}(z), \cdots, A_{k-1}(z)$ be analytic functions in $\Delta$ such that $i\left(A_{0}\right)=p$. If

$$
\max \left\{i\left(A_{j}\right): j=1, \cdots, k-1\right\}<p
$$

or

$$
\max \left\{\rho_{p}\left(A_{j}\right): j=1, \cdots, k-1\right\}<\rho_{p}\left(A_{0}\right),
$$

then every solution $f \not \equiv 0$ of equation (1.1) satisfies $i(f)=p+1$ and $\rho_{p}(f)=\infty$, $\rho_{p}\left(A_{0}\right) \leq \rho_{p+1}(f)=\rho_{M, p+1}(f) \leq \max \left\{\rho_{M, p}\left(A_{j}\right): j=0,1, \cdots, k-1\right\}$.

Lemma 2.4 [3] Let $f$ and $g$ be meromorphic functions in the unit disc $\Delta$ such that $0<\rho_{p}(f), \rho_{p}(g)<\infty$ and $0<\tau_{p}(f), \tau_{p}(g)<\infty$. Then we have

(i) If $\rho_{p}(f)>\rho_{p}(g)$, then we obtain

$$
\tau_{p}(f+g)=\tau_{p}(f g)=\tau_{p}(f) .
$$

(ii) If $\rho_{p}(f)=\rho_{p}(g)$ and $\tau_{p}(f) \neq \tau_{p}(g)$, then we get

$$
\rho_{p}(f+g)=\rho_{p}(f g)=\rho_{p}(f)=\rho_{p}(g) .
$$

Lemma 2.5 ([14]) Let $f$ be a meromorphic function in the unit disc and let $k \in \mathbb{N}$. Then

$$
m\left(r, \frac{f^{(k)}}{f}\right)=S(r, f),
$$

where $S(r, f)=O\left(\log ^{+} T(r, f)+\log \left(\frac{1}{1-r}\right)\right)$, possibly outside a set $E_{1} \subset[0,1)$ with $\int_{E_{1}} \frac{d r}{1-r}<\infty$. If $f$ is of finite order of growth, then

$$
m\left(r, \frac{f^{(k)}}{f}\right)=O\left(\log \left(\frac{1}{1-r}\right)\right) .
$$

Lemma 2.6 [2] Let $f$ be a meromorphic function in the unit disc for which $i(f)=$ $p \geq 1$ and $\rho_{p}(f)=\beta<\infty$, and let $k \in \mathbb{N}$. Then for any $\varepsilon>0$,

$$
m\left(r, \frac{f^{(k)}}{f}\right)=O\left(\exp _{p-2}\left(\log \frac{1}{1-r}\right)^{\beta+\varepsilon}\right)
$$

for all $r$ outside a set $E_{2} \subset[0,1)$ with $\int_{E_{2}} \frac{d r}{1-r}<\infty$. 
Lemma 2.7 Let $f$ be a meromorphic function in $\Delta$ with iterated order $\rho_{p}(f)=\rho$ $(0<\rho<\infty)$ and iterated type $\tau_{p}(f)=\tau(0<\tau<\infty)$. Then for any given $\beta<\tau$, there exists a subset $E_{3}$ of $[0,1)$ that has an infinite logarithmic measure such that $\log _{p-1} T(r, f)>\beta\left(\frac{1}{1-r}\right)^{\rho}$ holds for all $r \in E_{3}$.

Proof. When $p=1$, the lemma is proved in [21]. Thus we assume $p \geq 2$. By definitions of iterated order and iterated type, there exists an increasing sequence $\left\{r_{m}\right\}_{m=1}^{\infty} \subset[0,1)\left(r_{m} \rightarrow 1^{-}\right)$satisfying $\frac{1}{m}+\left(1-\frac{1}{m}\right) r_{m}<r_{m+1}$ and

$$
\lim _{m \rightarrow \infty} \frac{\log _{p-1} T\left(r_{m}, f\right)}{\left(\frac{1}{1-r_{m}}\right)^{\rho}}=\tau_{p}(f) .
$$

Then there exists a positive integer $m_{0}$ such that for all $m>m_{0}$ and for any given $0<\varepsilon<\tau_{p}(f)-\beta$, we have

$$
\log _{p-1} T\left(r_{m}, f\right)>\left(\tau_{p}(f)-\beta\right)\left(\frac{1}{1-r_{m}}\right)^{\rho} .
$$

For any given $\beta<\tau_{p}(f)-\varepsilon$, there exists a positive integer $m_{1}$ such that for all $m>m_{1}$ we have

$$
\left(1-\frac{1}{m}\right)^{\rho}>\frac{\beta}{\tau_{p}(f)-\varepsilon}
$$

Take $m \geq m_{2}=\max \left\{m_{0}, m_{1}\right\}$. By (2.2) and (2.3), for any $r \in\left[r_{m}, \frac{1}{m}+\left(1-\frac{1}{m}\right) r_{m}\right]$, we have

$$
\begin{aligned}
\log _{p-1} T(r, f) & \geq \log _{p-1} T\left(r_{m}, f\right)>\left(\tau_{p}(f)-\beta\right)\left(\frac{1}{1-r_{m}}\right)^{\rho} \\
& \geq\left(\tau_{p}(f)-\beta\right)\left(1-\frac{1}{m}\right)^{\rho}\left(\frac{1}{1-r}\right)^{\rho}>\beta\left(\frac{1}{1-r}\right)^{\rho} .
\end{aligned}
$$

Set $E_{3}=\cup_{m=m_{2}}^{\infty}\left[r_{m}, \frac{1}{m}+\left(1-\frac{1}{m}\right) r_{m}\right]$, then there holds

$$
m_{l} E_{3}=\sum_{m=m_{2}}^{\infty} \int_{r_{m}}^{\frac{1}{m}+\left(1-\frac{1}{m}\right) r_{m}} \frac{d t}{1-t}=\sum_{m=m_{2}}^{\infty} \log \frac{m}{m-1}=\infty .
$$

Lemma 2.8 [16] Let $f$ be a solution of equation (1.1) where the coefficients $A_{j}(z)(j=0, \cdots, k-1)$ are analytic functions in the disc $\Delta_{R}=\{z \in \mathbb{C}:|z|<R\}$, $0<R \leq \infty$. Let $n_{c} \in\{1, \cdots, k\}$ be the number of nonzero coefficients $A_{j}(z)$ $(j=0, \cdots, k-1)$, and let $\theta \in\left[0,2 \pi\left[\right.\right.$ and $\varepsilon>0$. If $z_{\theta}=\nu e^{i \theta} \in \Delta_{R}$ is such that $A_{j}\left(z_{\theta}\right) \neq 0$ for some $j=0, \cdots, k-1$, then for all $\nu<r<R$,

$$
\left|f\left(r e^{i \theta}\right)\right| \leq C \exp \left(n_{c} \int_{\nu}^{r} \max _{j=0, \cdots, k-1}\left|A_{j}\left(t e^{i \theta}\right)\right|^{\frac{1}{k-j}} d t\right)
$$


where $C>0$ is a constant satisfying

$$
C \leq(1+\varepsilon) \max _{j=0, \cdots, k-1}\left(\frac{\left|f^{(j)}\left(z_{\theta}\right)\right|}{\left(n_{c}\right)^{j} \max _{n=0, \cdots, k-1}\left|A_{n}\left(z_{\theta}\right)\right|^{\frac{j}{k-n}}}\right) .
$$

Lemma $2.9[1,14]$ Let $g:(0,1) \rightarrow \mathbb{R}$ and $h:(0,1) \rightarrow \mathbb{R}$ be monotone increasing functions such that $g(r) \leq h(r)$ holds outside of an exceptional set $E_{4} \subset[0,1)$ for which $\int_{E_{4}} \frac{d r}{1-r}<\infty$. Then there exists a constant $d \in(0,1)$ such that if $s(r)=$ $1-d(1-r)$, then $g(r) \leq h(s(r))$ for all $r \in[0,1)$.

Lemma 2.10 Let $A_{1}(z)$ and $A_{0}(z)$ be analytic functions in $\Delta$ such that $\rho_{p}\left(A_{0}\right)=\rho$ $(0<\rho<\infty), \tau_{p}\left(A_{0}\right)=\tau(0<\tau<\infty)$, and let $\rho_{p}\left(A_{1}\right)<\rho_{p}\left(A_{0}\right)$ and $\tau_{p}\left(A_{1}\right)<$ $\tau_{p}\left(A_{0}\right)$ if $\rho_{p}\left(A_{1}\right)=\rho_{p}\left(A_{0}\right)$. If $f \not \equiv 0$ is a solution of $(1.8)$ then $\rho_{p}(f)=\infty$, $\rho_{p+1}(f)=\rho_{p}\left(A_{0}\right)$ if $p>1$, while

$$
\rho_{p}(f)=\infty, \rho_{p}\left(A_{0}\right) \leq \rho_{p+1}(f) \leq \max \left\{\rho_{M}\left(A_{j}\right),(j=0,1)\right\}
$$

if $p=1$.

Proof. If $\rho_{p}\left(A_{1}\right)<\rho_{p}\left(A_{0}\right)$ then the result can easily deduced by Lemma 2.3. We prove only the case when $\rho_{p}\left(A_{0}\right)=\rho_{p}\left(A_{1}\right)=\rho$ and $\tau_{p}\left(A_{1}\right)<\tau_{p}\left(A_{0}\right)$. Since $f \not \equiv 0$, then by (1.8) we have

$$
A_{0}=-\left(\frac{f^{\prime \prime}}{f}+A_{1} \frac{f^{\prime}}{f}\right)
$$

Suppose that $f$ is of finite $p$-iterated order, then by Lemma 2.6

$$
T\left(r, A_{0}\right) \leq T\left(r, A_{1}\right)+O\left(\exp _{p-2}\left(\log \frac{1}{1-r}\right)^{\beta+\varepsilon}\right) \quad\left(\rho_{p}(f)=\beta<\infty\right)
$$

which implies the contradiction

$$
\tau_{p}\left(A_{0}\right) \leq \tau_{p}\left(A_{1}\right)
$$

Hence $\rho_{p}(f)=\infty$. By using inequality (2.4), we have

$$
\rho_{p+1}(f) \leq \max \left\{\rho_{p}\left(A_{1}\right), \rho_{p}\left(A_{0}\right)\right\}=\rho_{p}\left(A_{0}\right) .
$$

On the other hand, by Lemma 2.5

$$
T\left(r, A_{0}\right) \leq T\left(r, A_{1}\right)+O\left(\log ^{+} T(r, f)+\log \frac{1}{1-r}\right)
$$

holds possibly outside a set $E_{1} \subset[0,1)$ with $\int_{E_{1}} \frac{d r}{1-r}<\infty$. By $\tau_{p}\left(A_{1}\right)<\tau_{p}\left(A_{0}\right)$ we choose $\alpha_{0}, \alpha_{1}$ satisfying $\tau_{p}\left(A_{1}\right)<\alpha_{1}<\alpha_{0}<\tau_{p}\left(A_{0}\right)$ such that for $r \rightarrow 1^{-}$, we have

$$
T\left(r, A_{1}\right) \leq \exp _{p-1}\left\{\alpha_{1}\left(\frac{1}{1-r}\right)^{\rho}\right\} \text {. }
$$


By Lemma 2.7, there exists a subset $E_{2} \subset[0,1)$ of infinite logarithmic measure such that

$$
T\left(r, A_{0}\right)>\exp _{p-1}\left\{\alpha_{0}\left(\frac{1}{1-r}\right)^{\rho}\right\} .
$$

By $(2.8)-(2.10)$ we obtain for all $r \in E_{2}-E_{1}$

$$
(1-o(1)) \exp _{p-1}\left\{\alpha_{0}\left(\frac{1}{1-r}\right)^{\rho}\right\} \leq O\left(\log ^{+} T(r, f)+\log \frac{1}{1-r}\right) .
$$

By using (2.11) and Lemma 2.9, we obtain

$$
\rho_{p+1}(f) \geq \rho_{p}\left(A_{0}\right) .
$$

From $(2.7)$ and $(2.12)$ we get $\rho_{p}(f)=\infty$ and $\rho_{p+1}(f)=\rho_{p}\left(A_{0}\right)$.

\section{Proof of the Theorems and the Corollaries}

Proof of Theorem 1.1 Suppose that $f$ is an infinite iterated $p$-ordder meromorphic solution in $\Delta$ of (1.1). By (1.1), we have

$$
f^{(k)}=-\sum_{i=0}^{k-1} A_{i} f^{(i)}
$$

which implies

$$
\begin{gathered}
g_{k}=d_{k} f^{(k)}+d_{k-1} f^{(k-1)}+\cdots+d_{0} f \\
=\sum_{i=0}^{k-1}\left(d_{i}-d_{k} A_{i}\right) f^{(i)} .
\end{gathered}
$$

We can write $(3.2)$ as

$$
g_{k}=\sum_{i=0}^{k-1} \alpha_{i, 0} f^{(i)}
$$

where $\alpha_{i, 0}$ are defined in (1.4). Differentiating both sides of equation (3.3) and replacing $f^{(k)}$ with $f^{(k)}=-\sum_{i=0}^{k-1} A_{i} f^{(i)}$, we obtain

$$
\begin{aligned}
g_{k}^{\prime}= & \sum_{i=0}^{k-1} \alpha_{i, 0}^{\prime} f^{(i)}+\sum_{i=0}^{k-1} \alpha_{i, 0} f^{(i+1)}=\sum_{i=0}^{k-1} \alpha_{i, 0}^{\prime} f^{(i)}+\sum_{i=1}^{k} \alpha_{i-1,0} f^{(i)} \\
& =\alpha_{0,0}^{\prime} f+\sum_{i=1}^{k-1} \alpha_{i, 0}^{\prime} f^{(i)}+\sum_{i=1}^{k-1} \alpha_{i-1,0} f^{(i)}+\alpha_{k-1,0} f^{(k)} \\
= & \alpha_{0,0}^{\prime} f+\sum_{i=1}^{k-1} \alpha_{i, 0}^{\prime} f^{(i)}+\sum_{i=1}^{k-1} \alpha_{i-1,0} f^{(i)}-\sum_{i=0}^{k-1} \alpha_{k-1,0} A_{i} f^{(i)}
\end{aligned}
$$




$$
=\left(\alpha_{0,0}^{\prime}-\alpha_{k-1,0} A_{0}\right) f+\sum_{i=1}^{k-1}\left(\alpha_{i, 0}^{\prime}+\alpha_{i-1,0}-\alpha_{k-1,0} A_{i}\right) f^{(i)} .
$$

We can rewrite $(3.4)$ as

$$
g_{k}^{\prime}=\sum_{i=0}^{k-1} \alpha_{i, 1} f^{(i)}
$$

where

$$
\alpha_{i, 1}=\left\{\begin{array}{c}
\alpha_{i, 0}^{\prime}+\alpha_{i-1,0}-\alpha_{k-1,0} A_{i}, \text { for all } i=1, \cdots, k-1 \\
\alpha_{0,0}^{\prime}-A_{0} \alpha_{k-1,0}, \text { for } i=0
\end{array}\right.
$$

Differentiating both sides of equation (3.5) and replacing $f^{(k)}$ with $f^{(k)}=-\sum_{i=0}^{k-1} A_{i} f^{(i)}$, we obtain

$$
\begin{gathered}
g_{k}^{\prime \prime}=\sum_{i=0}^{k-1} \alpha_{i, 1}^{\prime} f^{(i)}+\sum_{i=0}^{k-1} \alpha_{i, 1} f^{(i+1)}=\sum_{i=0}^{k-1} \alpha_{i, 1}^{\prime} f^{(i)}+\sum_{i=1}^{k} \alpha_{i-1,1} f^{(i)} \\
=\alpha_{0,1}^{\prime} f+\sum_{i=1}^{k-1} \alpha_{i, 1}^{\prime} f^{(i)}+\sum_{i=1}^{k-1} \alpha_{i-1,1} f^{(i)}+\alpha_{k-1,1} f^{(k)} \\
=\alpha_{0,1}^{\prime} f+\sum_{i=1}^{k-1} \alpha_{i, 1}^{\prime} f^{(i)}+\sum_{i=1}^{k-1} \alpha_{i-1,1} f^{(i)}-\sum_{i=0}^{k-1} A_{i} \alpha_{k-1,1} f^{(i)} \\
=\left(\alpha_{0,1}^{\prime}-\alpha_{k-1,1} A_{0}\right) f+\sum_{i=1}^{k-1}\left(\alpha_{i, 1}^{\prime}+\alpha_{i-1,1}-A_{i} \alpha_{k-1,1}\right) f^{(i)}
\end{gathered}
$$

which implies that

$$
g_{k}^{\prime \prime}=\sum_{i=0}^{k-1} \alpha_{i, 2} f^{(i)}
$$

where

$$
\alpha_{i, 2}=\left\{\begin{array}{c}
\alpha_{i, 1}^{\prime}+\alpha_{i-1,1}-A_{i} \alpha_{k-1,1}, \text { for all } i=1, \cdots, k-1 \\
\alpha_{0,1}^{\prime}-A_{0} \alpha_{k-1,1}, \text { for } i=0
\end{array}\right.
$$

By using the same method as above we can easily deduce that

$$
g_{f}^{(j)}=\sum_{i=0}^{k-1} \alpha_{i, j} f^{(i)}, j=0,1, \cdots, k-1,
$$

where

$$
\alpha_{i, j}=\left\{\begin{array}{c}
\alpha_{i, j-1}^{\prime}+\alpha_{i-1, j-1}-A_{i} \alpha_{k-1, j-1}, \text { for all } i=1, \cdots, k-1 \\
\alpha_{0, j-1}^{\prime}-A_{0} \alpha_{k-1, j-1}, \text { for } i=0
\end{array}\right.
$$


and

$$
\alpha_{i, 0}=d_{i}-d_{k} A_{i}, \text { for all } i=0,1, \cdots, k-1 .
$$

By $(3.3)-(3.12)$ we obtain the system of equations

$$
\left\{\begin{array}{c}
g_{k}=\alpha_{0,0} f+\alpha_{1,0} f^{\prime}+\cdots+\alpha_{k-1,0} f^{(k-1)} \\
g_{k}^{\prime}=\alpha_{0,1} f+\alpha_{1,1} f^{\prime}+\cdots+\alpha_{k-1,1} f^{(k-1)} \\
g_{k}^{\prime \prime}=\alpha_{0,2} f+\alpha_{1,2} f^{\prime}+\cdots+\alpha_{k-1,2} f^{(k-1)} \\
\cdots \\
g_{k}^{(k-1)}=\alpha_{0, k-1} f+\alpha_{1, k-1} f^{\prime}+\cdots+\alpha_{k-1, k-1} f^{(k-1)}
\end{array}\right.
$$

By Cramer's rule, and since $h_{k} \not \equiv 0$ we have

$$
f=\frac{\left|\begin{array}{ccccc}
g_{k} & \alpha_{1,0} & . & . & \alpha_{k-1,0} \\
g_{k}^{\prime} & \alpha_{1,1} & . & \cdot & \alpha_{k-1,1} \\
\cdot & \cdot & \cdot & \cdot & \cdot \\
\cdot & \cdot & \cdot & \cdot & \cdot \\
g_{k}^{(k-1)} & \alpha_{1, k-1} & \cdot & \cdot & \alpha_{k-1, k-1}
\end{array}\right|}{h}
$$

Then

$$
f=C_{0} g_{k}+C_{1} g_{k}^{\prime}+\cdots+C_{k-1} g_{k}^{(k-1)},
$$

where $C_{j}$ are finite iterated $p$-order meromorphic functions in $\Delta$ depending on $\alpha_{i, j}$, where $\alpha_{i, j}$ is defined in (3.11).

If $\rho_{p}\left(g_{k}\right)<+\infty$, then by (3.15) we obtain $\rho_{p}(f)<+\infty$, and this is a contradiction. Hence $\rho_{p}\left(g_{k}\right)=\rho_{p}(f)=+\infty$.

Now, we prove that $\rho_{p+1}\left(g_{k}\right)=\rho_{p+1}(f)=\rho$. By $(3.2)$, we get $\rho_{p+1}\left(g_{k}\right) \leq$ $\rho_{p+1}(f)$ and by $(3.15)$ we have $\rho_{p+1}(f) \leq \rho_{p+1}\left(g_{k}\right)$. This yield $\rho_{p+1}\left(g_{k}\right)=\rho_{p+1}(f)=$ $\rho$.

Furthermore, if $f$ is a finite iterated $p$-order meromorphic solution in $\Delta$ of equation (1.1) such that

$$
\rho_{p}(f)>\max \left\{\rho_{p}\left(A_{i}\right)(i=0, \cdots, k-1), \rho_{p}\left(d_{j}\right) \quad(j=0,1, \cdots, k)\right\},
$$

then

$$
\rho_{p}(f)>\max \left\{\rho_{p}\left(\alpha_{i, j}\right): i=0, \cdots, k-1, j=0, \cdots, k-1\right\} .
$$

By (3.2) and (3.16) we have $\rho_{p}\left(g_{k}\right) \leq \rho_{p}(f)$. Now, we prove $\rho_{p}\left(g_{k}\right)=\rho_{p}(f)$. If $\rho_{p}\left(g_{k}\right)<\rho_{p}(f)$, then by $(3.15)$ and (3.17) we get

$$
\rho_{p}(f) \leq \max \left\{\rho_{p}\left(C_{j}\right)(j=0, \cdots, k-1), \rho_{p}\left(g_{k}\right)\right\}<\rho_{p}(f)
$$

and this is a contradiction. Hence $\rho_{p}\left(g_{k}\right)=\rho_{p}(f)$.

Remark 3.1 From (3.13), it follows that the condition $h \not \equiv 0$ is equivalent to the condition $g_{k}, g_{k}^{\prime}, \cdots, g_{k}^{(k-1)}$ are linearly independent over the field of meromorphic functions of finite iterated $p$-order. 
Proof of Theorem 1.2 Suppose that $f$ is an infinite iterated $p$-order meromorphic solution in $\Delta$ of equation (1.1) with $\rho_{p+1}(f)=\rho$. Set $w(z)=g_{k}-\varphi$. Since $\rho_{p}(\varphi)<\infty$, then by Theorem 1.1 we have $\rho_{p}(w)=\rho_{p}\left(g_{k}\right)=\infty$ and $\rho_{p+1}(w)=\rho_{p+1}\left(g_{k}\right)=\rho$. To prove $\bar{\lambda}_{p}\left(g_{k}-\varphi\right)=\lambda_{p}\left(g_{k}-\varphi\right)=\infty$ and $\bar{\lambda}_{p+1}\left(g_{k}-\varphi\right)=\lambda_{p+1}\left(g_{k}-\varphi\right)=\rho$ we need to prove $\bar{\lambda}_{p}(w)=\lambda_{p}(w)=\infty$ and $\bar{\lambda}_{p+1}(w)=\lambda_{p+1}(w)=\rho$. By $g_{k}=w+\varphi$, and using (3.15), we get

$$
f=C_{0} w+C_{1} w^{\prime}+\cdots+C_{k-1} w^{(k-1)}+\psi_{k}(z),
$$

where

$$
\psi_{k}(z)=C_{0} \varphi+C_{1} \varphi^{\prime}+\cdots+C_{k-1} \varphi^{(k-1)} .
$$

Substituting (3.18) into (1.1), we obtain

$$
C_{k-1} w^{(2 k-1)}+\sum_{j=0}^{2 k-2} \phi_{j} w^{(j)}=-\left(\psi_{k}^{(k)}+A_{k-1}(z) \psi_{k}^{(k-1)}+\cdots+A(z) \psi_{k}\right)=H
$$

where $C_{k-1}, \phi_{j}(j=0, \cdots, 2 k-1)$ are meromorphic functions in $\Delta$ with finite iterated $p$-order. Since $\psi_{k}(z)$ is not a solution of (1.1), it follows that $H \not \equiv 0$. Then by Lemma 2.2, we obtain $\bar{\lambda}_{p}(w)=\lambda_{p}(w)=\infty$ and $\bar{\lambda}_{p+1}(w)=\lambda_{p+1}(w)=\rho$, i. e., $\bar{\lambda}_{p}\left(g_{k}-\varphi\right)=\lambda_{p}\left(g_{k}-\varphi\right)=\infty$ and $\bar{\lambda}_{p+1}\left(g_{k}-\varphi\right)=\lambda_{p+1}\left(g_{k}-\varphi\right)=\rho$.

Suppose that $f$ is a finite iterated $p$-order meromorphic solution in $\Delta$ of equation (1.1) such that (1.6) holds. Set $w(z)=g_{k}-\varphi$. Since $\rho_{p}(\varphi)<\rho_{p}(f)$, then by Theorem 1.1 we have $\rho_{p}(w)=\rho_{p}\left(g_{k}\right)=\rho_{p}(f)$. To prove $\bar{\lambda}_{p}\left(g_{k}-\varphi\right)=\lambda_{p}\left(g_{k}-\varphi\right)=\rho_{p}(f)$ we need to prove $\bar{\lambda}_{p}(w)=\lambda_{p}(w)=\rho_{p}(f)$. Using the same reasoning as above, we get

$$
C_{k-1} w^{(2 k-1)}+\sum_{j=0}^{2 k-2} \phi_{j} w^{(j)}=-\left(\psi_{k}^{(k)}+A_{k-1}(z) \psi_{k}^{(k-1)}+\cdots+A(z) \psi_{k}\right)=F
$$

where $C_{k-1}, \phi_{j}(j=0, \cdots, 2 k-1)$ are meromorphic functions in $\Delta$ with finite iterated $p$-order $\rho_{p}\left(C_{k-1}\right)<\rho_{p}(f), \rho_{p}\left(\phi_{j}\right)<\rho_{p}(f)(j=0, \cdots, 2 k-1)$, and

$$
\psi_{k}(z)=C_{0} \varphi+C_{1} \varphi^{\prime}+\cdots+C_{k-1} \varphi^{(k-1)}, \rho_{p}(F)<\rho_{p}(f) .
$$

Since $\psi_{k}(z)$ is not a solution of (1.1), it follows that $F \not \equiv 0$. Then by Lemma 2.1, we obtain $\bar{\lambda}_{p}(w)=\lambda_{p}(w)=\rho_{p}(f)$, i. e., $\bar{\lambda}_{p}\left(g_{k}-\varphi\right)=\lambda_{p}\left(g_{k}-\varphi\right)=\rho_{p}(f)$.

Proof of Corollary 1.3 Suppose that $f$ is a nontrivial solution of (1.8). Then by Lemma 2.3, we have

$$
\rho_{p}\left(A_{0}\right) \leq \rho_{p+1}(f) \leq \max \left\{\rho_{M, p}\left(A_{j}\right) \quad(j=0,1)\right\} \quad(p \geq 1) .
$$

By the same reasoning as before we obtain that

$$
\left\{\begin{array}{l}
g_{2}=\alpha_{0,0} f+\alpha_{1,0} f^{\prime} \\
g_{2}^{\prime}=\alpha_{0,1} f+\alpha_{1,1} f^{\prime}
\end{array}\right.
$$


where

$$
\alpha_{0,0}=d_{0}-d_{2} A_{0}, \alpha_{1,1}=d_{2} A_{1}^{2}-\left(d_{2} A_{1}\right)^{\prime}-d_{1} A_{1}-d_{2} A_{0}+d_{0}+d_{1}^{\prime}
$$

and

$$
\alpha_{0,1}=d_{2} A_{0} A_{1}-\left(d_{2} A_{0}\right)^{\prime}-d_{1} A_{0}+d_{0}^{\prime}, \alpha_{1,0}=d_{1}-d_{2} A_{1} .
$$

First, we suppose that $d_{2} \not \equiv 0$. We have

$$
\begin{gathered}
h_{2}=\left|\begin{array}{cc}
\alpha_{1,0} & \alpha_{0,0} \\
\alpha_{1,1} & \alpha_{0,1}
\end{array}\right| \\
=-d_{2}^{2} A_{0}^{2}-d_{0} d_{2} A_{1}^{2}+\left(-d_{2} d_{1}+d_{1}^{\prime} d_{2}+2 d_{0} d_{2}-d_{1}^{2}\right) A_{0} \\
+\left(d_{2}^{\prime} d_{0}-d_{2} d_{0}^{\prime}+d_{0} d_{1}\right) A_{1}+d_{1} d_{2} A_{0} A_{1}-d_{1} d_{2} A_{0}^{\prime}+d_{0} d_{2} A_{1}^{\prime} \\
+d_{2}^{2} A_{0}^{\prime} A_{1}-d_{2}^{2} A_{0} A_{1}^{\prime}+d_{0}^{\prime} d_{1}-d_{0} d_{1}^{\prime}-d_{0}^{2} .
\end{gathered}
$$

By $d_{2} \not \equiv 0, A_{0} \not \equiv 0$ and Lemma 2.4, we have $\rho_{p}(h)=\rho_{p}\left(A_{0}\right)$. Hence $h \not \equiv 0$. Now suppose $d_{2} \equiv 0, d_{1} \not \equiv 0$ or $d_{2} \equiv 0, d_{1} \equiv 0$ and $d_{0} \not \equiv 0$, then by using a similar reasoning as above we get $h \not \equiv 0$, and we obtain

$$
f=\frac{\alpha_{1,0} g_{f}^{\prime}-\alpha_{1,1} g_{f}}{h_{2}}
$$

It is clear that $\rho_{p}\left(g_{2}\right) \leq \rho_{p}(f)\left(\rho_{p+1}\left(g_{2}\right) \leq \rho_{p+1}(f)\right)$ and by (3.21) we have $\rho_{p}(f) \leq$ $\rho_{p}\left(g_{2}\right)\left(\rho_{p+1}(f) \leq \rho_{p+1}\left(g_{2}\right)\right)$. Hence $\rho_{p}\left(g_{2}\right)=\rho_{p}(f)\left(\rho_{p+1}\left(g_{2}\right)=\rho_{p+1}(f)\right)$.

Proof of Corollary 1.4 Set $w(z)=d_{2} f^{\prime \prime}+d_{1} f^{\prime}+d_{0} f-\varphi$. Then, by $\rho_{p}(\varphi)<\infty$, we have $\rho_{p}(w)=\rho_{p}\left(g_{2}\right)=\rho_{p}(f)$ and $\rho_{p+1}(w)=\rho_{p+1}\left(g_{2}\right)=\rho_{p+1}(f)$. In order to prove $\bar{\lambda}_{p+1}\left(g_{2}-\varphi\right)=\lambda_{p+1}\left(g_{2}-\varphi\right)=\rho_{p+1}(f)$, we need to prove only $\bar{\lambda}_{p+1}(w)=$ $\lambda_{p+1}(w)=\rho_{p+1}(f)$. Using $g_{2}=w+\varphi$, we get from (3.21)

$$
f=\frac{\alpha_{1,0} w^{\prime}-\alpha_{1,1} w}{h_{2}}+\psi_{2}
$$

where

$$
\psi_{2}(z)=\frac{\alpha_{1,0} \varphi^{\prime}-\alpha_{1,1} \varphi}{h_{2}}
$$

Substituting (3.22) into equation (1.8), we obtain

$$
\begin{gathered}
\frac{\alpha_{1,0}}{h_{2}} w^{\prime \prime \prime}+\phi_{2} w^{\prime \prime}+\phi_{1} w^{\prime}+\phi_{0} w \\
=-\left(\psi_{2}^{\prime \prime}+A_{1}(z) \psi_{2}^{\prime}+A_{0}(z) \psi_{2}\right)=A,
\end{gathered}
$$

where $\phi_{j}(j=0,1,2)$ are meromorphic functions in $\Delta$ with $\rho_{p}\left(\phi_{j}\right)<\infty(j=0,1,2)$. First, we prove that $\psi_{2} \not \equiv 0$. Suppose that $\psi_{2} \equiv 0$, then by (3.23) we obtain

$$
\alpha_{1,1}=\alpha_{1,0} \frac{\varphi^{\prime}}{\varphi}
$$


It follows that by using Lemma 2.6

$$
m\left(r, \alpha_{1,1}\right) \leq m\left(r, \alpha_{1,0}\right)+O\left(\exp _{p-2}\left(\log \frac{1}{1-r}\right)^{\beta+\varepsilon}\right), \quad \rho_{p}(\varphi)=\beta<\infty .
$$

(i) If $d_{2} \not \equiv 0$, then by using Lemma 2.4 we obtain the contradiction

$$
\left\{\begin{array}{l}
\rho_{p}\left(A_{0}\right) \leq \rho_{p}\left(A_{1}\right), \text { if } \rho_{p}\left(A_{0}\right)>\rho_{p}\left(A_{1}\right) \\
\tau_{p}\left(A_{0}\right) \leq \tau_{p}\left(A_{1}\right), \text { if } \rho_{p}\left(A_{0}\right)=\rho_{p}\left(A_{1}\right)
\end{array}\right.
$$

(ii) If $d_{2} \equiv 0$ and $d_{1} \not \equiv 0$, we obtain the contradiction

$$
\rho_{p}\left(A_{1}\right) \leq \rho_{p}\left(d_{1}\right)
$$

(iii) If $d_{2}=d_{1} \equiv 0$ and $d_{0} \not \equiv 0$, we have by $(3.25)$

$$
d_{0}=\alpha_{1,1}=\alpha_{1,0} \frac{\varphi^{\prime}}{\varphi}=0 \times \frac{\varphi^{\prime}}{\varphi} \equiv 0
$$

which is a contradiction. Hence $\psi_{2} \not \equiv 0$. It is clear now that $\psi_{2} \not \equiv 0$ cannot be a solution of (1.8) because $\rho_{p}\left(\psi_{2}\right)<\infty$. Then, by Lemma 2.1 , we obtain $\bar{\lambda}_{p+1}\left(g_{2}-\varphi\right)=$ $\lambda_{p+1}\left(g_{2}-\varphi\right)=\rho_{p+1}(f)$, i. e., $\bar{\lambda}_{p+1}(w)=\lambda_{p+1}(w)=\rho_{p+1}(f)$.

\section{References}

[1] S. Bank, General theorem concerning the growth of solutions of first-order algebraic differential equations, Compositio Math. 25 (1972), 61-70.

[2] B. Belaïdi, Oscillation of fast growing solutions of linear differential equations in the unit disc, Acta Univ. Sapientiae Math. 2 (2010), no. 1, 25-38.

[3] B. Belaïdi, A. El Farissi, Fixed points and iterated order of differential polynomial generated by solutions of linear differential equations in the unit disc, J. Adv. Res. Pure Math. 3 (2011), no. 1, 161-172.

[4] L. G. Bernal, On growth $k$-order of solutions of a complex homogeneous linear differential equation, Proc. Amer. Math. Soc. 101 (1987), no. 2, 317-322.

[5] T. B. Cao and H. X. Yi, The growth of solutions of linear differential equations with coefficients of iterated order in the unit disc, J. Math. Anal. Appl. 319 (2006), no. 1, 278-294.

[6] T. B. Cao, The growth, oscillation and fixed points of solutions of complex linear differential equations in the unit disc, J. Math. Anal. Appl. 352 (2009), no. 2, 739-748.

[7] T. B. Cao, H. Y. Xu and C. X. Zhu, On the complex oscillation of differential polynomials generated by meromorphic solutions of differential equations in the unit disc, Proc. Indian Acad. Sci. Math. Sci. 120 (2010), no. 4, 481-493. 
[8] T. B. Cao and Z. S. Deng, Solutions of non-homogeneous linear differential equations in the unit disc, Ann. Polo. Math. 97(2010), no. 1, 51-61.

[9] T. B. Cao, L. M. Li, J. Tu and H. Y. Xu, Complex oscillation of differential polynomials generated by analytic solutions of differential equations in the unit disc, Math. Commun. 16 (2011), no. 1, 205-214.

[10] Z. X. Chen and K. H. Shon, The growth of solutions of differential equations with coefficients of small growth in the disc, J. Math. Anal. Appl. 297 (2004), no. 1, $285-304$.

[11] I. E. Chyzhykov, G. G. Gundersen and J. Heittokangas, Linear differential equations and logarithmic derivative estimates, Proc. London Math. Soc. (3) 86 (2003), no. 3, 735-754.

[12] A. El Farissi, B. Belaïdi and Z. Latreuch, Growth and oscillation of differential polynomials in the unit disc, Electron. J. Diff. Equ., Vol. 2010(2010), No. 87, 1-7.

[13] W. K. Hayman, Meromorphic functions, Oxford Mathematical Monographs Clarendon Press, Oxford, 1964.

[14] J. Heittokangas, On complex differential equations in the unit disc, Ann. Acad. Sci. Fenn. Math. Diss. 122 (2000), 1-54.

[15] J. Heittokangas, R. Korhonen and J. Rättyä, Fast growing solutions of linear differential equations in the unit disc, Results Math. 49 (2006), no. 3-4, 265-278.

[16] J. Heittokangas, R. Korhonen and J. Rättyä, Growth estimates for solutions of linear complex differential equations, Ann. Acad. Sci. Fenn. Math. 29 (2004), no. $1,233-246$.

[17] L. Kinnunen, Linear differential equations with solutions of finite iterated order, Southeast Asian Bull. Math. 22 (1998), no. 4, 385-405.

[18] I. Laine, Nevanlinna theory and complex differential equations, de Gruyter Studies in Mathematics, 15. Walter de Gruyter \& Co., Berlin-New York, 1993.

[19] I. Laine and J. Rieppo, Differential polynomials generated by linear differential equations, Complex Var. Theory Appl. 49 (2004), no. 12, 897-911.

[20] I. Laine, Complex differential equations, Handbook of differential equations: ordinary differential equations. Vol. IV, 269-363, Handb. Differ. Equ., Elsevier/North-Holland, Amsterdam, 2008.

[21] Z. Latreuch, B. Belaïdi and Abdallah El Farissi, Complex oscillation of differential polynomials in the unit disc, Period. Math. Hungar. 66 (2013), no. 1, 45-60.

[22] Z. Latreuch and B. Belaïdi, Growth and oscillation of differential polynomials generated by complex differential equations, Electron. J. Diff. Equ., Vol. 2013 (2013), No. 16, 1-14. 
[23] Z. Latreuch and B. Belaïdi, Properties of solutions of complex differential equations in the unit disc, International Journal of Analysis and Applications, Volume 4, Number 2 (2014), 159-173.

[24] Z. Latreuch and B. Belaïdi, Further results on the properties of differential polynomials generated by solutions of complex differential equations, Submitted.

[25] M. Tsuji, Potential Theory in Modern Function Theory, Chelsea, New York, (1975), reprint of the 1959 edition.

DOI: $10.7862 /$ rf.2014.7

Zinelâabidine Latreuch

email: z.latreuch@gmail.com

Benharrat Belaïdi - Corresponding author

email: belaidi@univ-mosta.dz

Department of Mathematics

Laboratory of Pure and Applied Mathematics

University of Mostaganem (UMAB)

B. P. 227 Mostaganem-(Algeria)

Received 10.09.2013, Accepted 20.11.2013 